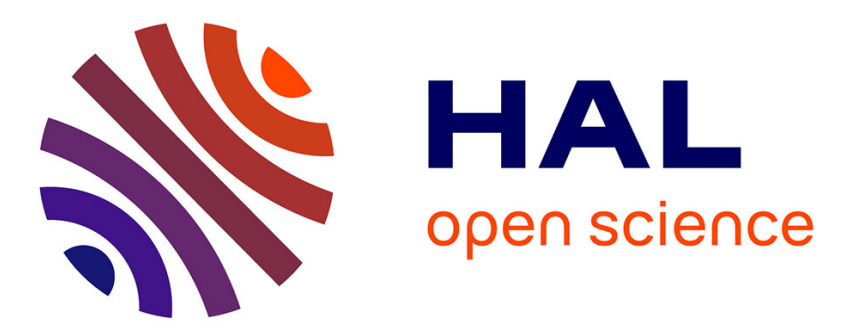

\title{
List of keywords for polymer science (IUPAC Technical Report)
}

Stanislaw Slomkowski, Christopher Fellows, Roger C. Hiorns, Richard Jones, Przemyslaw Kubisa, Christine Luscombe, Tamaki Nakano, Gregory Russell, Claudio dos Santos, Carmen Scholz, et al.

\section{To cite this version:}

Stanislaw Slomkowski, Christopher Fellows, Roger C. Hiorns, Richard Jones, Przemyslaw Kubisa, et al. List of keywords for polymer science (IUPAC Technical Report). Pure and Applied Chemistry, 2019, 91 (6), pp.997-1027. 10.1515/pac-2018-0917 . hal-02430791

\section{HAL Id: hal-02430791 \\ https: / hal-univ-pau.archives-ouvertes.fr/hal-02430791}

Submitted on 27 Nov 2020

HAL is a multi-disciplinary open access archive for the deposit and dissemination of scientific research documents, whether they are published or not. The documents may come from teaching and research institutions in France or abroad, or from public or private research centers.
L'archive ouverte pluridisciplinaire HAL, est destinée au dépôt et à la diffusion de documents scientifiques de niveau recherche, publiés ou non, émanant des établissements d'enseignement et de recherche français ou étrangers, des laboratoires publics ou privés. 


\title{
IUPAC Technical Report
}

Stanislaw Slomkowski*, Christopher M. Fellows, Roger C. Hiorns, Richard G. Jones, Przemyslaw Kubisa, Christine K. Luscombe, Tamaki Nakano, Gregory T. Russell, Claudio G. dos Santos, Carmen Scholz, Natalie Stingelin and Michael G. Walter

\section{List of keywords for polymer science (IUPAC Technical Report)}

https://doi.org/10.1515/pac-2018-0917

Received September 24, 2018; accepted February 21, 2019

\begin{abstract}
This paper provides a list of the most important terms from all areas of polymer science including polymer chemistry, polymer physics, polymer technology and polymer properties. These have been assembled into a representative list of terms that serves as an IUPAC recommended list of keywords for polymer science.
\end{abstract}

Keywords: IUPAC; keyword; polymer science.

\section{CONTENTS}

1 INTRODUCTION

2 LIST OF KEYWORDS ARRANGED INTO CATEGORIES

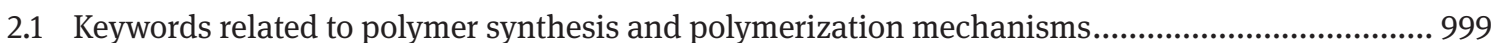

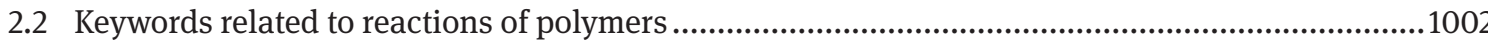

2.3 Keywords related to polymer class names and common polymer names ......................................1003

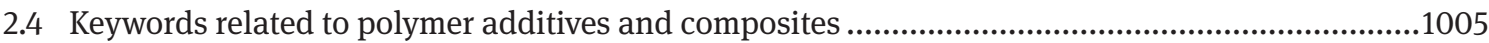

2.5 Keywords related to polymer structure, processing, properties and applications ......................... 1006

2.6 Keywords related to polymer characterization methods .................................................................. 1012

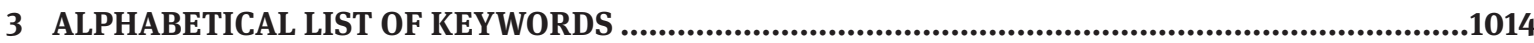

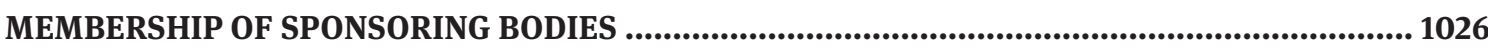

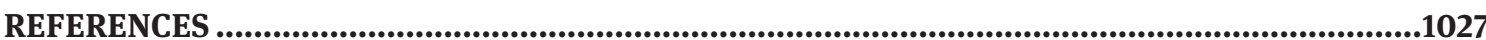

Article note: Sponsoring body: IUPAC Polymer Division and the IUPAC Subcommittee on Polymer Terminology.

*Corresponding author: Stanislaw Slomkowski, Center of Molecular and Macromolecular Studies, Polish Academy of Sciences, Sienkiewicza 112, 90-363 Lodz, Poland, e-mail: staslomk@cbmm.lodz.pl

Christopher M. Fellows: University of New England, School of Science and Technology, Armidale, NSW 2351, Australia

Roger C. Hiorns: CNRS/Univ Pau and Pays Adour, Institut des Science Analytiques et Physico-Chimie pour l'Environnement et les Materiaux, UMR 5254, 64000 Pau, France

Richard G. Jones: Functional Materials Group, School of Physical Sciences, University of Kent, Canterbury CT2 7NH, UK Przemyslaw Kubisa: Center of Molecular and Macromolecular Studies, Polish Academy of Sciences, Sienkiewicza 112, 90-363 Lodz, Poland

Christine K. Luscombe: University of Washington, Department of Materials Science and Engineering, Seattle, WA 98195, USA Tamaki Nakano: Institute for Catalysis, Hokkaido University, Sapporo, Hokkaido, Japan

Gregory T. Russell: School of Physical and Chemical Sciences, University of Canterbury, Private Bag 4800, Christchurch, New Zealand

Claudio G. dos Santos: Univ. Fed. Ouro Preto, Dept. Quim, BR-35400000 Ouro Preto, MG, Brazil

Carmen Scholz: University of Alabama in Huntsville, Department of Chemistry, Huntsville, AL 35899, USA

Natalie Stingelin: Georgia Institute of Technology, School of Materials Science and Engineering, Atlanta, GA 30332, USA Michael G. Walter: University of North Carolina - Charlotte, Department of Chemistry, 9201 University City, Blvd, Charlotte, NC 28223, USA 


\section{Introduction}

Internet searches of scientific literature return results based mainly on electronic engines, which work to identify relevant keywords. These keywords are supplied by the authors of publications. The selection of keywords that best describe the content of a publication is therefore very important. Some publishers of journals related to polymer science provide a recommended list of keywords from which authors must select the most relevant terms. Wiley, for example does this [1]. Some publishers, on the other hand, give authors license to provide their own keywords, while others provide a non-binding list of keywords, i.e. authors may use it or ignore it as they prefer. In addition to these variations, different authors place a different emphasis on the selection of keywords. Some take it as a serious exercise and strive to provide keywords that genuinely encapsulate the contents of their article, while others regard keywords as a nuisance and do not particularly care if they are a "low precision" guide to the content of their publication. Furthermore, as science is a dynamic discipline, newly discovered phenomena are constantly giving rise to new terms that may not be included in existing lists of keywords. All these factors introduce a degree of ambiguity which, in turn, makes searching the literature by keywords less accurate.

For these reasons it is worthwhile to collect the most salient terms from all areas of polymer science, i.e. polymer chemistry, polymer physics, polymer technology, and polymer properties, and then to assemble these terms into a representative list that may serve as an IUPAC-recommended list of keywords for polymer science. This is the aim of this paper. Ideally, all polymer-related journals would employ this list and would insist on authors selecting from it, except where terms are newly coined, in which event - if prudent - they would be added to the list, making it an evolving document. This would ultimately result in a streamlined search tool: faster, more comprehensive, and more accurate.

The following list was created by a task-group of the IUPAC Polymer Division, which collected the most relevant terms, identified on the basis of:

a. terms from IUPAC Polymer Division recommendations given in the IUPAC Compendium of Polymer Terminology and Nomenclature (the "Purple Book") [2];

b. lists of keywords for Wiley journals [1] (J. Polym. Sci. A Polym. Chem. [3], J. Polym. Sci. B Polym. Phys. [4], J. Appl. Polym. Sci. [5], Polym. Int. [6], Macromol. Chem. Phys. [7]);

c. titles of chapters and sections in the Encyclopedia of Polymer Science and Technology [8], Comprehensive Polymer Science [9], Ulmann's Encyclopedia of Industrial Chemistry [10];

d. terms that appeared as keywords in the most cited (usually more than 500 citations) polymer-related papers (on the basis of ISI Web of Knowledge [11]);

e. terms that appeared as keywords in publications in Prog. Polym. Sci. [12] in the period 2008-2015; and

f. terms from the Polymer Science Dictionary [13].

The list was divided into six categories and entries were arranged alphabetically within them, with categories for keywords related to: polymer synthesis and polymerization mechanisms; reactions of polymers; polymer class names and common polymer names; polymer additives and composites; polymer structure, processing, properties and applications; and polymer characterization methods. In addition, the complete alphabetical list is included at the end of this paper. Where appropriate, authors may choose to insert their own polymerspecific keyword. This should be done in accord with recent IUPAC nomenclature recommendations $[14,15]$.

The generation of new keywords was avoided where possible in order to avoid confusion; however, where new keywords are required, it is recommended that they conform to definitions given in the IUPAC Compendium of Polymer Terminology and Nomenclature (the "Purple Book"). The spellings of American English are recommended as these are more commonly used, and for searching efficiency it is obviously preferable if workers adhere to the forms of a particular variant of English. It is also recommended that the singular form be used. 


\title{
2 List of keywords arranged into categories
}

\subsection{Keywords related to polymer synthesis and polymerization mechanisms}

\author{
activated monomer \\ activation energy \\ activator generated by electron transfer atom transfer radical polymerization (AGET-ATRP) \\ activator regenerated by electron transfer atom transfer radical polymerization (ARGET-ATRP) \\ acyclic diene metathesis polymerization (ADMET) \\ anionic polymerization \\ anionic ring-opening polymerization \\ asymmetric polymerization \\ atom transfer radical polymerization (ATRP) \\ branching \\ bulk polymerization
}

catalyst

catalyst transfer polymerization (CTP)

cationic polymerization

cationic ring-opening polymerization

chain polymerization

chain scission

chain transfer

click reaction

cobalt-mediated radical polymerization

condensative chain polymerization (CCP)

controlled anionic polymerization

controlled cationic polymerization

controlled ionic polymerization

controlled polymerization

controlled radical polymerization (CRP)

convergent approach

coordination polymer

coordination polymerization

coordination ring-opening polymerization

copolymerization

coupling

crosslinking

cyclization

degenerative chain transfer radical polymerization

degradation

degree of polymerization

depolymerization

dispersion polymerization

divergent approach 


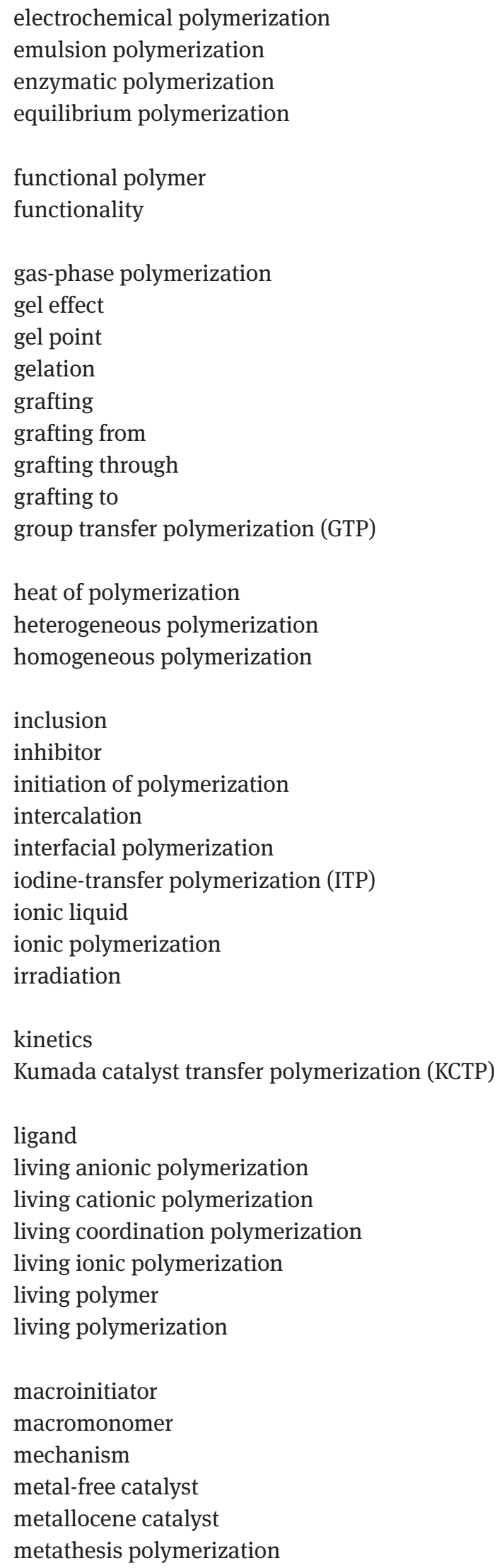


microemulsion polymerization

miniemulsion polymerization

molar mass

molar mass dispersity

nitroxide mediated polymerization

olefin polymerization catalyst

organobismuthine-mediated radical polymerization (BIRP)

organometallic-mediated radical polymerization (OMRP)

organostibane-mediated radical polymerization (SBRP)

organotellurium-mediated radical polymerization (TERP)

oxidative polymerization

photoinitiated polymerization

photopolymerization

plasma polymerization

polyaddition

polycondensation

post-metallocene catalyst

precipitation polymerization

prepolymer

propagation

pseudo-ionic polymerization

quantum chemistry

radiation polymerization

radical polymerization

rate of polymerization

reactive injection molding

reactivity ratio (in copolymerization)

reverse atom transfer radical polymerization (reverse ATRP)

reverse iodine-transfer polymerization (RITP)

reversible-addition-fragmentation chain-transfer polymerization (RAFT)

reversible addition-fragmentation radical polymerization (RAFRP)

reversible deactivation anionic polymerization (RDAP)

reversible deactivation cationic polymerization (RDCP)

reversible deactivation coordination polymerization (RDCP)

reversible deactivation ionic polymerization (RDIP)

reversible deactivation polymerization (RDP)

reversible deactivation radical polymerization (RDRP)

reversible polymerization

ring-opening metathesis polymerization (ROMP)

ring-opening polymerization

sequential polymerization

sol-gel

solid-phase synthesis

solid-state polymerization 
solution polymerization

stable radical mediated polymerization

stereospecific polymerization

surface-initiated polymerization

suspension polymerization

telomerization

template

template polymerization

termination

thermodynamics

thiocarbonyl-mediated radical polymerization (TMRP)

transition-metal-mediated radical polymerization

Ziegler-Natta catalyst

Ziegler-Natta polymerization

\title{
2.2 Keywords related to reactions of polymers
}

\author{
aminolysis \\ biodegradation \\ chemical modification \\ composting \\ conjugate \\ conjugation \\ degradation \\ enzymatic degradation \\ functionalization \\ hydrolysis \\ metabolization \\ modification \\ molecular recognition \\ oxidation \\ PEGylation \\ photodegradation \\ post-polymerization functionalization \\ pyrolysis \\ reactive processing \\ recycling
}


thermal degradation

tissue engineering

vulcanization

\subsection{Keywords related to polymer class names and common polymer names}

acrylic polymer

aliphatic polyester

aromatic polyester

alternating copolymer

amphiphilic polymer

aramid

biomaterial

biomedical polymer

biopolymer

carbohydrate

cellulose

chitin

chitosan

collagen

copolymer

cyclodextrin

dendrimer

dendritic polymer

dextran

epoxy

fluoropolymer

gelatin

graphene

hydrophilic polymer

hydrophobic polymer

hyperbranched polymer

inorganic polymer

ion-exchange polymer

ionic polymer

ionomer

ladder

metal-containing

metal-organic

nanocomposite 
natural rubber

nylon

oligomer

organic-inorganic hybrid material

polyacetylene

polyacrylate

polyacrylamide

polyacrylonitrile

polyamide

polyaniline

polycarbonate

polyelectrolyte

polyester

poly(ester amide)

polyether

polyethylene

polyfluorene

polyhydroxyalkanoate

polyimide

polyisobutylene

polylactide

polymer electrolyte

polymethacrylate

polyolefin

poly(oxirane)

polyphenylene

poly(phenylene diamine)

poly(phenylene vinylene)

polyphosphazene

polypropylene

polypyrrole

polyrotaxane

polysaccharide

polysilane

polysiloxane

polystyrene

polythiophene

polyurea

polyurethane

poly(vinyl alcohol)

poly(vinyl chloride)

poly(vinyl ether)

preceramic polymer

protein

random copolymer

reinforced polymer

renewable resource 
responsive polymer

rubber

semiconducting polymer shape memory polymer silicon polymer silsesquioxane smart polymer star polymer starch statistical copolymer superabsorbent polymer supramolecular polymer

thermoplastic elastomer thermoplastic polymer thermoset thermosetting polymer

unsaturated polyester

water soluble polymer

\title{
2.4 Keywords related to polymer additives and composites
}

\author{
acid neutralizer \\ additive \\ adhesion promotor \\ adhesive \\ alumina \\ anti-blocking agent \\ anti-fogging agent \\ anti-microbial additive \\ antioxidant \\ antistatic agent \\ blowing agent \\ carbon black \\ carbon fiber \\ carbon nanotube \\ catalyst deactivator \\ catalyst quencher \\ clay \\ colorant \\ compatibilizer \\ coupling agent \\ crosslinking agent
}




\author{
defoaming agent \\ degradation promoter \\ dispersing agent \\ drug \\ dye \\ filler \\ flame retardant \\ foaming agent \\ fullerene \\ glass fiber \\ graphene \\ graphene oxide \\ heat stabilizer \\ impact modifier \\ light stabilizer \\ lubricant \\ mold release agent \\ montmorillonite \\ nanotube \\ nucleation agent \\ optical brightener \\ pigment \\ plasticizer \\ processing aid \\ reinforcement \\ scavenger \\ stabilizer \\ surfactant
}

\title{
2.5 Keywords related to polymer structure, processing, properties and applications
}

\author{
ablation \\ adhesion \\ adsorbent \\ adsorption \\ aggregate \\ aggregation \\ aging
}




\author{
alpha transition \\ amorphous polymer \\ anisotropy \\ annealing \\ aspect ratio \\ association \\ atactic \\ barrier property \\ beta transition \\ bioadhesion \\ biocompatible polymer \\ biodegradable polymer \\ biological application \\ biomedical application \\ biomimetic polymer \\ birefringence \\ blend \\ block copolymer \\ branched polymer \\ brittle \\ brush
}

bulk-heterojunction (BHJ)

casting

cellular

charge transfer

charge transport

chiral

coating

coil

colloid

comb

compatibility

composite

compost

compression

conducting polymer

configuration

conformation

controlled release

conjugated polymer

core-shell

crack

craze

crazing

creep

critical solution temperature

crosslink

crystal structure 


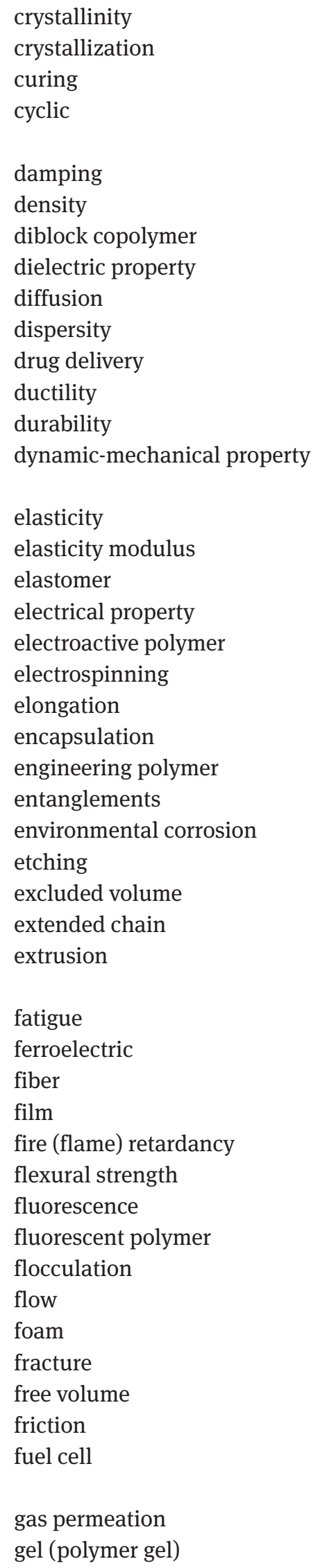




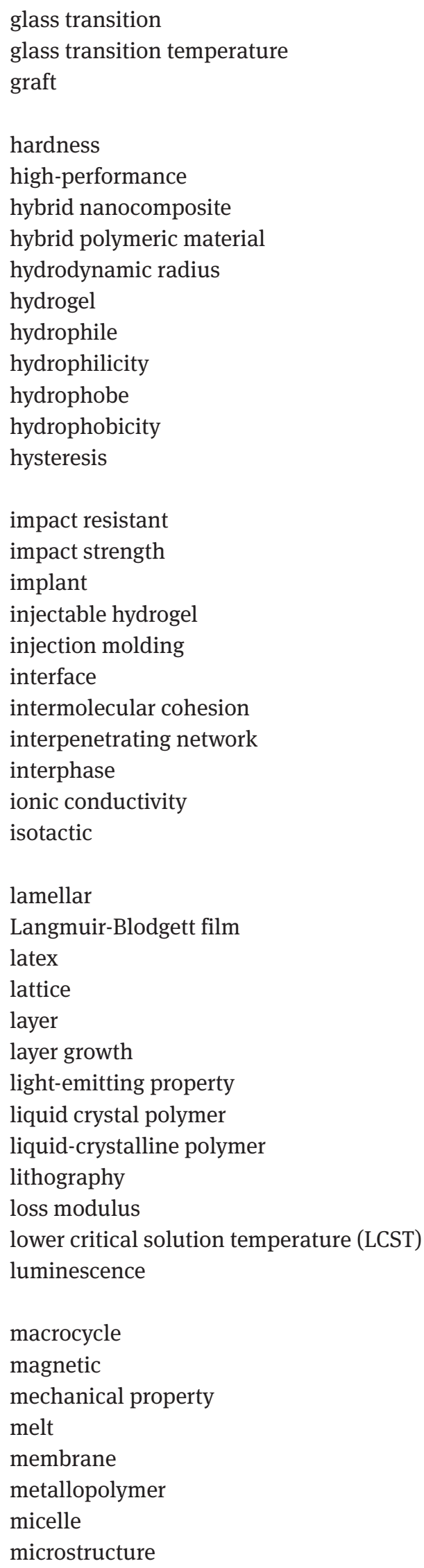


miscibility

mixed binary brush

mixing

modulus

molding

molecular dynamics

molecularly imprinted polymer

monolayer

morphology

nanofiber

nanofiller

nanofoam

nanogel

nanoparticle

nanostructure

network

non-linear optical property

nucleation

optical anisotropy

optical property

optical activity

organic electronics

organic field-effect transistor (OFET)

organic light-emitting diode (OLED)

organic photovoltaics (OPVs)

orientation

$\pi$-conjugated polymer

permanent network

pharmacological application

phase behavior

phase separation

phase transition

phosphorescence

photoactive polymer

photonic crystal

photoresist

photosensitive polymer

photovoltaic application

piezoelectric

polarization

polyanion

polycation

polymer brush

polymer film

polymer light emitting diode (PLED)

polymer melt 


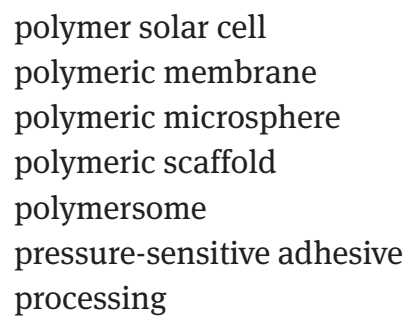


vesicle

viscoelastic property

viscosity

wear

weathering

yield strength (yielding)

Young modulus

\subsection{Keywords related to polymer characterization methods}

atomic force microscopy (AFM)

calorimetry

capillary electrophoresis

chemical corrosion

chromatography

circular dichroism (CD)

cloud point determination

computer simulation

conductometry

cyclic voltammetry (CV)

degree of swelling

dielectric spectroscopy

differential scanning calorimetry (DSC)

differential thermal analysis (DTA)

dilatometry

dynamic light scattering (DLS)

dynamic-mechanical analysis (DMA)

dynamic-mechanical thermal analysis (DMTA)

electron diffraction

electron paramagnetic spin resonance (EPR or ESR)

electrophoresis

electrospray ionization (ESI)

field-flow fractionation (FFF)

fourier transform infrared (FTIR) spectroscopy

fractionation

fracture analysis

gel permeation chromatography (GPC)

impact resistance

impact test

implant testing

infrared (IR) spectroscopy

light scattering 


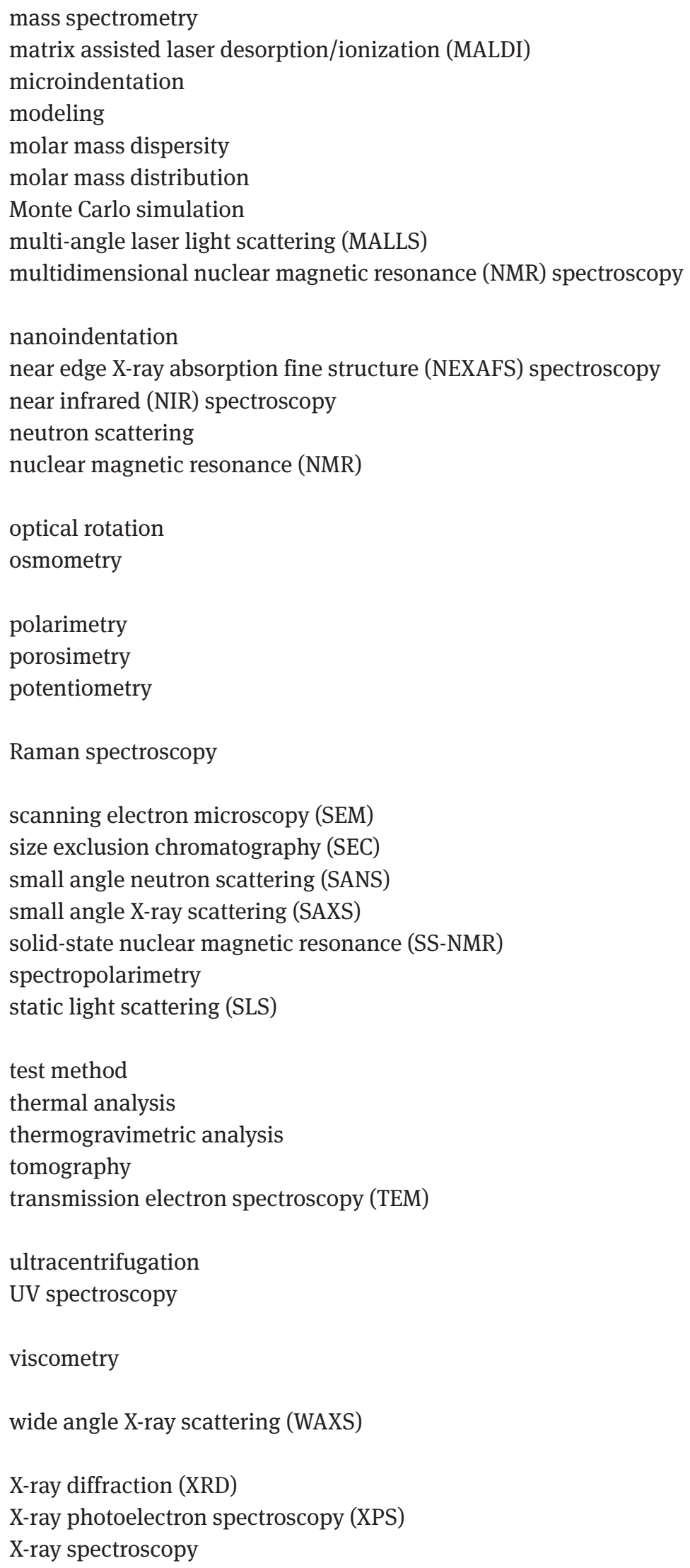




\section{Alphabetical list of keywords}

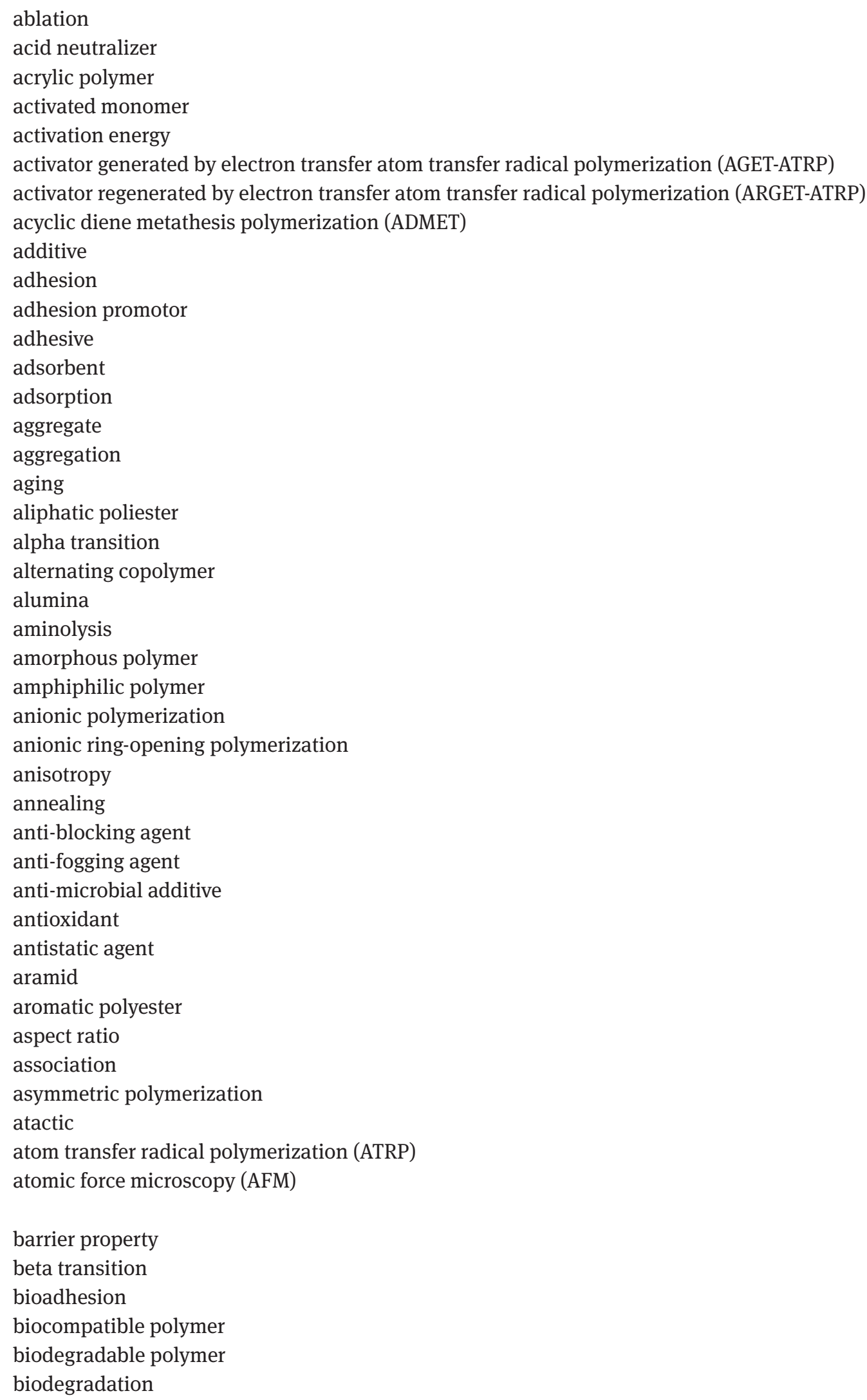


biological application

biomaterial

biomedical application

biomedical polymer

biomimetic polymer

biopolymer

birefringence

blend

block copolymer

blowing agent

branched polymer

branching

brittle

brush

bulk polymerization

bulk-heterojunction (BHJ)

calorimetry

capillary electrophoresis

carbohydrate

carbon black

carbon fiber

carbon nanotube

casting

catalyst

catalyst deactivator

catalyst quencher

catalyst transfer polymerization (CTP)

cationic polymerization

cationic ring-opening polymerization

cellular

cellulose

chain polymerization

chain scission

chain transfer

charge transfer

charge transport

chemical corrosion

chemical modification

chiral

chitin

chitosan

chromatography

circular dichroism (CD)

clay

click reaction

cloud point determination

coating

cobalt-mediated radical polymerization

coil 


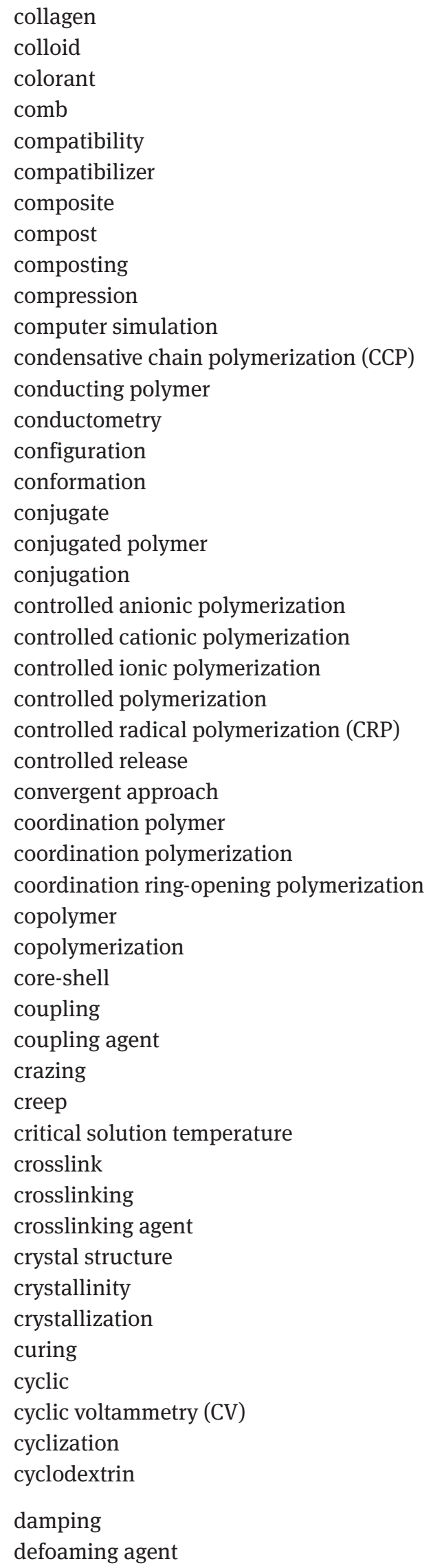


degenerative chain transfer radical polymerization

degradation

degradation promoter

degree of polymerization

degree of swelling

dendrimer

dendritic polymer

density

depolymerization

dextran

diblock copolymer

dielectric property

dielectric spectroscopy

differential scanning calorimetry (DSC)

differential thermal analysis (DTA)

diffusion

dilatometry

dispersing agent

dispersion polymerization

dispersity

divergent approach

drug

drug delivery

ductility

durability

dye

dynamic light scattering (DLS)

dynamic-mechanical analysis (DMA)

dynamic-mechanical property

dynamic-mechanical thermal analysis (DMTA)

elasticity

elasticity modulus

elastomer

electrical property

electroactive polymer

electrochemical polymerization

electron diffraction

electron paramagnetic spin resonance (EPR or ESR)

electrophoresis

electrospinning

electrospray ionization (ESI)

elongation

emulsion polymerization

encapsulation

engineering polymer

entanglements

environmental corrosion

enzymatic degradation

enzymatic polymerization 
epoxy

equilibrium polymerization

etching

excluded volume

extended chain

extrusion

fatigue

ferroelectric

fiber

field-flow fractionation (FFF)

filler

film

fire (flame) retardancy

flame retardant

flexural strength

flocculation

flow

fluorescence

fluorescent polymer

fluoropolymer

foam

foaming agent

fourier transform infrared (FTIR) spectroscopy

fractionation

fracture

fracture analysis

free volume

friction

fuel cell

fullerene

functional polymer

functionality

functionalization

gas permeation

gas-phase polymerization

gel (polymer gel)

gel effect

gel permeation chromatography (GPC)

gel point

gelatin

gelation

glass fiber

glass transition

glass transition temperature

graft

grafting

grafting from

grafting through 


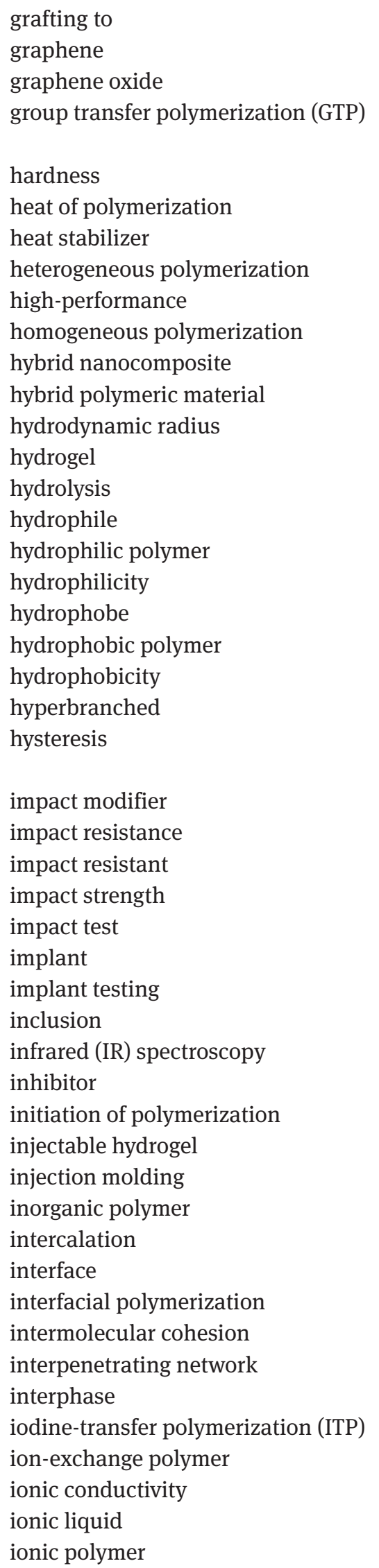


ionic polymerization

ionomer

irradiation

isotactic

kinetics

Kumada catalyst transfer polymerization (KCTP)

ladder

lamellar

Langmuir-Blodgett film

latex

lattice

layer

layer growth

ligand

light scattering

light-emitting property

light stabilizer

liquid crystal polymer

liquid-crystalline polymer

lithography

living anionic polymerization

living cationic polymerization

living coordination polymerization

living ionic polymerization

living polymer

living polymerization

loss modulus

lower critical solution temperature (LCST)

lubricant

luminescence

macrocycle

macroinitiator

macromonomer

magnetic

mass spectrometry

matrix assisted laser desorption/ionization (MALDI)

mechanical property

mechanism

melt

membrane

metabolization

metal-containing

metal-free catalyst

metal-organic

metallocene catalyst

metallopolymer 
metathesis polymerization

micelle

microemulsion polymerization

microindentation

microstructure

miniemulsion polymerization

miscibility

mixed binary brush

mixing

modeling

modification

modulus

molar mass

molar mass dispersity

molar mass distribution

mold release agent

molding

molecular dynamics

molecular recognition

molecularly imprinted polymer

monolayer

Monte Carlo simulation

montmorillonite

morphology

multi-angle laser light scattering (MALLS)

multidimensional nuclear magnetic resonance (NMR) spectroscopy

nanocomposite

nanofiber

nanofiller

nanofoam

nanogel

nanoindentation

nanoparticle

nanostructure

nanotube

natural rubber

near edge X-ray absorption fine structure (NEXAFS) spectroscopy

near infrared (NIR) spectroscopy

network

neutron scattering

nitroxide mediated polymerization

non-linear optical property

nuclear magnetic resonance (NMR)

nucleation

nucleation agent

nylon

olefin polymerization catalyst

oligomer 
optical anisotropy

optical brightener

optical property

optical rotation

organic electronics

organic field-effect transistor (OFET)

organic light-emitting diode (OLED)

organic photovoltaics (OPVs)

organic-inorganic hybrid material

organobismuthine-mediated radical polymerization (BIRP)

organometallic-mediated radical polymerization (OMRP)

organostibane-mediated radical polymerization (SBRP)

organotellurium-mediated radical polymerization (TERP)

orientation

osmometry

oxidation

oxidative polymerization

$\pi$-conjugated polymer

PEGylation

permanent network

phase behavior

phase separation

phase transition

phosphorescence

photoactive polymer

photodegradation

photoinitiated polymerization

photonic crystal

photopolymerization

photoresist

photosensitive polymer

photovoltaic application

piezoelectric

pigment

plasma polymerization

plasticizer

polyacetylene

polyacrylamide

polarimetry

polarization

polyacrylate

polyacrylonitrile

polyaddition

polyamide

polyaniline

polyanion

polycarbonate

polycation 


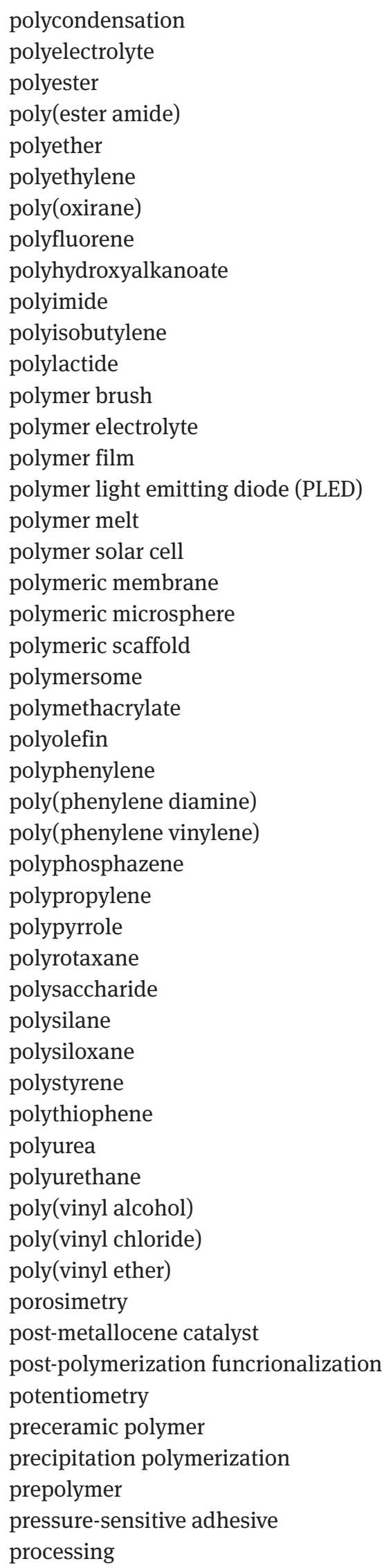




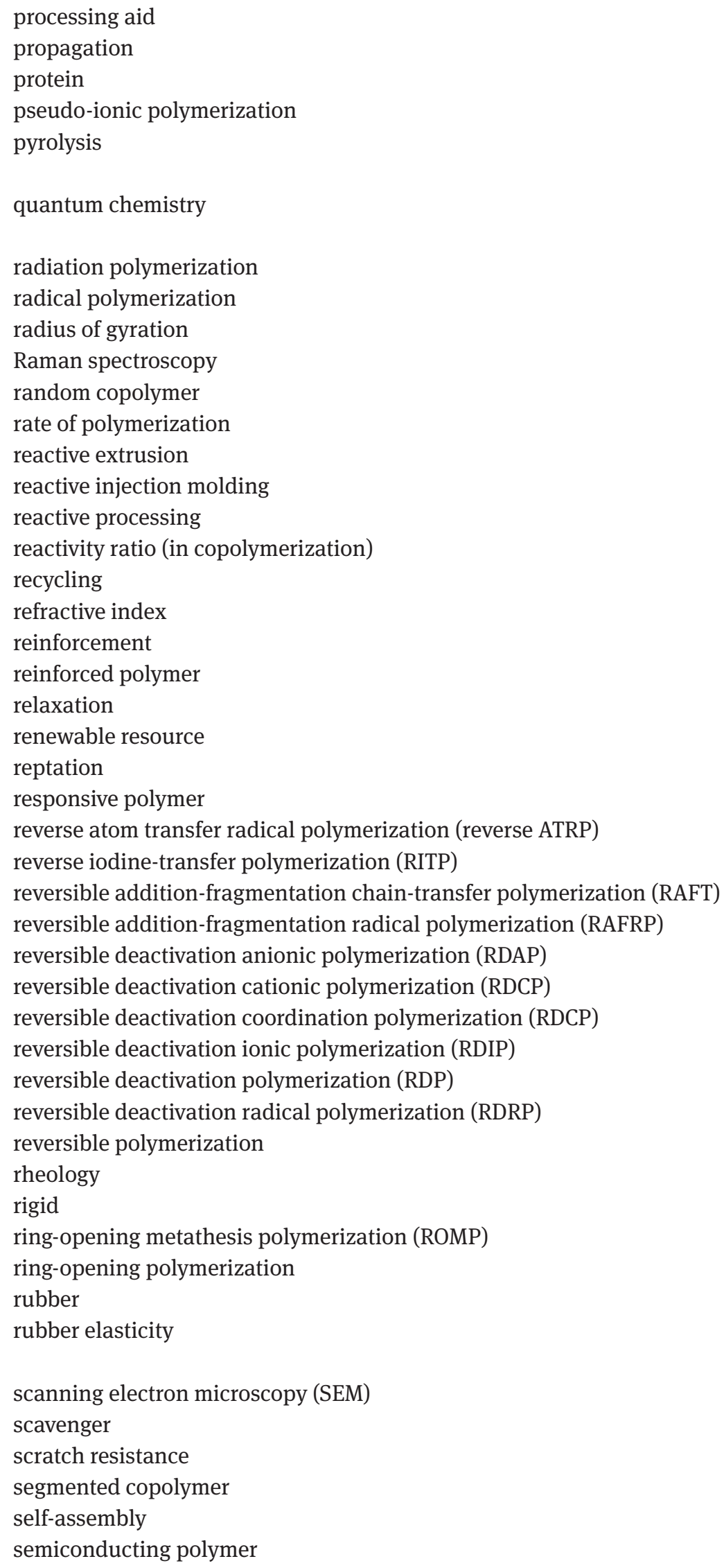




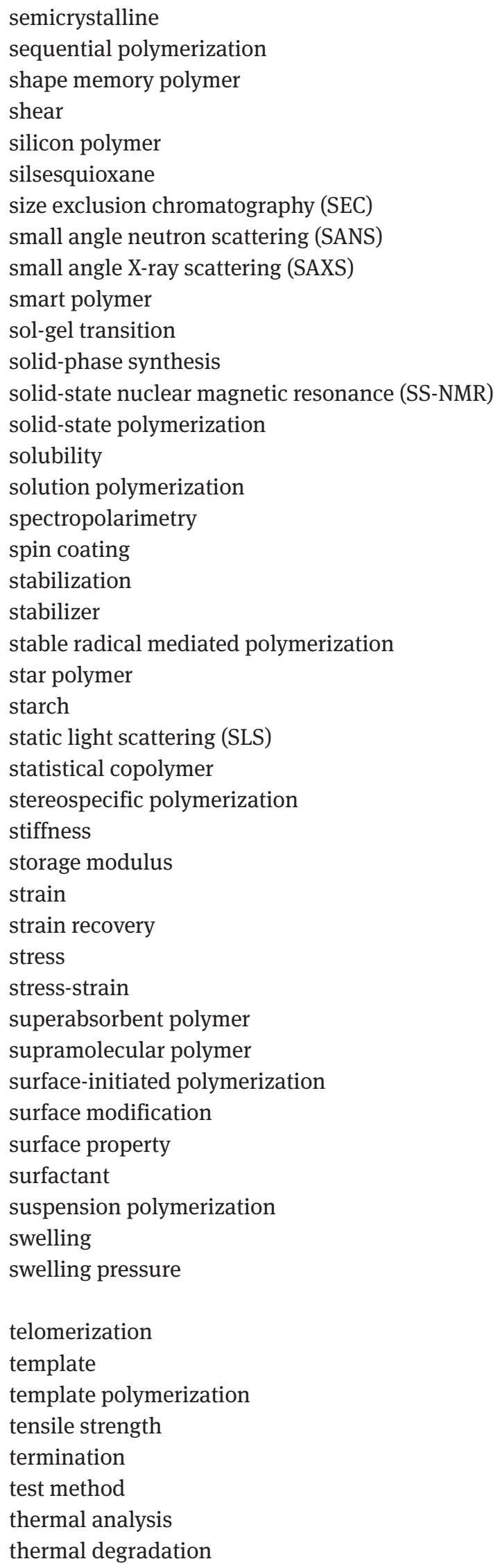




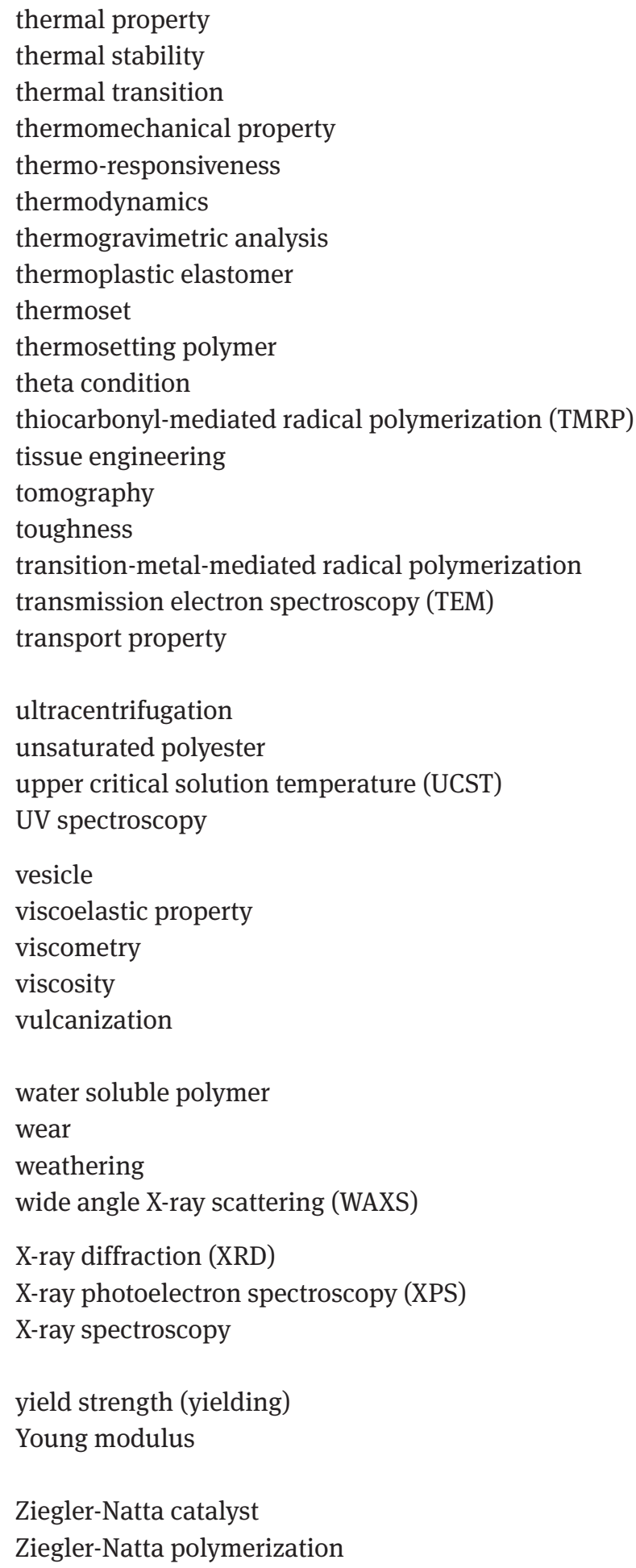

\section{Membership of sponsoring bodies}

Membership of the IUPAC Polymer Division Committee for the period 2016-2017 was as follows:

President: G. T. Russell (New Zealand); Vice President: C. K. Luscombe (USA); Secretary: M. G. Walter (USA); Past President: M. Buback (Germany); Titular Members: S. Beuermann (Germany); J. He (China); I. Lacík 
(Slovakia); M. Sawamoto (Japan); N. Stingelin (UK); Y. Yagci (Turkey); Associate Members: R. C. Hiorns (France); M. Hess (Germany); R. Hutchinson (Canada); G. Moad (Australia); R. Advincula (USA); D. Auhl (Netherlands); National Representatives: R. Adhikari (Nepal); M. C. H. Chan (Malaysia); C. G. dos Santos (Brazil); V. P. Hoven (Thailand); C.-S. Hsu (Taipei, China); R. G. Jones (UK); D. S. Lee (Korea); M. Malinconico (Italy); O. Philippova (Russia); J. Vohlídal (Czech Republic)

Membership of the Subcommittee on Polymer Terminology during the preparation of this document (20102017) was as follows: Chair: R. G. Jones (UK), 2006-2013; R. C. Hiorns (France), from 2014; Secretary: R. C. Hiorns (France), 2010-2013; C. K. Luscombe (USA), 2014-2015; P. D. Topham (UK), from 2016; Members: R. Adhikari (Nepal); G. Allegra (Italy); M. Barón (Argentina); R. Boucher (UK); P. Carbone (UK); M. C. H. Chan (Malaysia); T. Chang (Korea); J. Chen (USA); C. Fellows (Australia); A. Fradet (France); K. Hatada (Japan); J. He (China); K.-H. Hellwich (Germany); M. Hess (Germany); R. C. Hiorns (France); P. Hodge (UK);

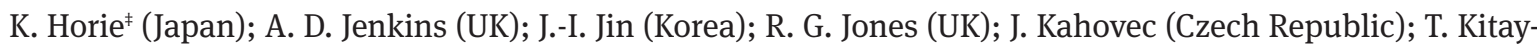
ama (Japan); P. Kratochvíl (Czech Republic); P. Kubisa (Poland); C. K. Luscombe (USA); S. V. Meille (Italy);

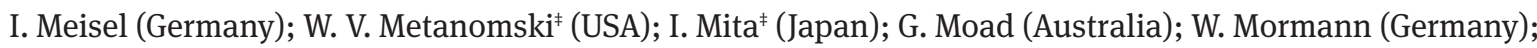
N. Nakabayashi (Japan); T. Nakano (Japan), C. K. Ober (USA); S. Penczek (Poland); O. Philippova (Russia); M. D. Purbrick (UK); G. Raos (Italy); L. P. Rebelo (Portugal); M. Rinaudo (France); G. T. Russell (New Zealand); C. G. dos Santos (Brazil); I. Schopov (Bulgaria); C. Scholz (USA); F. Schué (France); V. P. Shibaev (Russia); S. Słomkowski (Poland); D. W. Smith (USA), R. F. T. Stepto ${ }^{\ddagger}$ (UK); N. Stingelin (UK); D. Tabak (Brazil); P. Theato (Germany); P. D. Topham (UK); J.-P. Vairon (France); M. Vert (France); J. Vohlídal (Czech Republic); M. G. Walter (USA); E. S. Wilks (USA); W. J. Work (USA).

Acknowledgement: This manuscript (PAC-REP-18-09-17) was prepared in the framework of IUPAC project 2010-036-1-400.

\section{References}

[1] Keyword Catalogue, (Wiley-VCH) https://application.wiley-vch.de/vch/journals/keyword.php.

[2] IUPAC. Compendium of Polymer Terminology and Nomenclature (IUPAC Recommendations 2008) (the “Purple Book"). Prepared for publication by R. G. Jones, J. Kahovec, R. Stepto, E. S. Wilks, M. Hess, T. Kitayama, W. V. Metanomski, with advice from A. Jenkins and P. Kratochvíl, RSC Publishing, Cambridge, UK (2008).

[3] J. Polym. Sci. A Polym. Chem., http://onlinelibrary.wiley.com/journal/10.1002/(ISSN)1099-0518.

[4] J. Polym. Sci. B Polym. Phys., http://onlinelibrary.wiley.com/journal/10.1002/(ISSN)1099-0488.

[5] J. Appl. Polym. Sci., http://onlinelibrary.wiley.com/journal/10.1002/(ISSN)1097-4628.

[6] Polym. Int., http://onlinelibrary.wiley.com/journal/10.1002/(ISSN)1097-0126.

[7] Macromol. Chem. Phys., http://onlinelibrary.wiley.com/journal/10.1002/(ISSN)1521-3935.

[8] Encyclopedia of Polymer Science and Technology, http://onlinelibrary.wiley.com/book/10.1002/0471440264.

[9] Polymer Science: A Comprehensive Reference, Matyjaszewski K and Möller M (eds.), Elsevier BV, Amsterdam (2012). http://www.sciencedirect.com/science/referenceworks/9780080878621.

[10] Ullmann's Encyclopedia of Industrial Chemistry, Wiley-VCH, Weinheim (2011).

[11] ISI Web of Knowledge, http://webofscience.com.

[12] Prog. Polym. Sci., http://www.journals.elsevier.com/progress-in-polymer-science.

[13] Alger M. Polymer Science Dictionary, Springer, Dordrecht (2017).

[14] R. G. Jones, T. Kitayama, K.-H. Hellwich, M. Hess, A. D. Jenkins, J. Kahovec, P. Kratochvíl, I. Mita, W. Mormann, C. K. Ober, S. Penczek, R. F. T. Stepto, K. Thurlow, J. Vohlídal, E. S. Wilks. Pure Appl. Chem. 88, 1073 (2016).

[15] W. Mormann, K.-H. Hellwich, J. Chen, E. S. Wilks. Pure Appl. Chem. 89, 1695 (2017).

‡ Deceased. 